\title{
Paraneoplastic cerebellar degeneration associated with breast cancer: A case report and review of the literature
}

\author{
LANYUN YAN $^{1 *}$, XIN DONG $^{1 *}$, HUAN XU $^{1}$, JINGJING HUANG $^{2}$, \\ WEI WANG $^{1}$, LIN HUANG ${ }^{1}$, QI WAN ${ }^{1}$ and JIE GONG ${ }^{1}$ \\ ${ }^{1}$ Department of Neurology, The First Affiliated Hospital of Nanjing Medical University, Nanjing, Jiangsu 210029; \\ ${ }^{2}$ Department of Gerontology, The Second Affiliated Hospital of Nanjing Medical University, Nanjing, Jiangsu 210011, P.R. China
}

Received November 10, 2017; Accepted April 25, 2018

DOI: $10.3892 / \mathrm{mco} .2018 .1638$

\begin{abstract}
Paraneoplastic cerebellar degeneration (PCD) is a rare neurological complication of cancer characterized by rapid development of cerebellar ataxia. We herein present a case of a 67-year-old female patient with PCD caused by breast cancer. The patient presented with progressively worsening cerebellar deficits that had been misdiagnosed for several months prior to the identification of the anti-Yo autoantibodies in the serum. A whole-body positron emission tomography/computed tomography scan revealed a lesion in the lower outer quadrant of the left breast with slightly increased metabolism. On mammography, a lobulated high-density mass was identified in the left breast. The patient underwent left breast lumpectomy and the histological examination confirmed the presence of an invasive ductal carcinoma. After breast surgery, the patient exhibited marked neurological improvement at the 12-month follow-up. Therefore, it is crucial that clinicians include paraneoplastic neurological syndromes in the differential diagnosis of neurological disorders. The detection of characterized onconeural antibodies in the serum or cerebrospinal fluid may provide guidance in the search for an underlying tumor.
\end{abstract}

Correspondence to: Dr Jie Gong, Department of Neurology, The First Affiliated Hospital of Nanjing Medical University, 300 Guangzhou Road, Nanjing, Jiangsu 210029, P.R. China

E-mail: gong_jie@126.com

*Contributed equally

Abbreviations: PNS, paraneoplastic neurological syndromes; PCD, paraneoplastic cerebellar degeneration; CSF, cerebrospinal fluid; MRI, magnetic resonance imaging; PET/CT, positron emission tomography/computed tomography; FDG, fluorodeoxyglucose; SUVmax, maximum standardized uptake value

Key words: paraneoplastic neurological syndromes, breast cancer, paraneoplastic cerebellar degeneration, onconeural antibodies, anti-Yo

\section{Introduction}

Paraneoplastic neurological syndromes (PNS) are defined as remote effects of cancer involving the nervous system that are unrelated to the direct effects of the tumor and its metastasis, infection, ischemia or metabolic disturbance (1). PNS are fairly rare, affecting $<1 \%$ of patients with cancer, but may be severely debilitating due to the impairment of neurological functions (2). The diagnosis of PNS may be challenging, as they may affect any part of the nervous system and may mimic any other neurological disorder. Clinicians should consider PNS in the differential diagnosis of neurological disorders.

The occurrence of antibodies directed against onconeural antigens expressed by both the tumor cells and the nervous systems indicates that the disorders may be mediated by immunological mechanisms $(3,4)$. The possibility of the presence of an underlying tumor is highly associated with the type of antibodies identified (5). Detection of the characterized onconeural antibodies may suggest the diagnosis of a neurological disorder as paraneoplastic and guide the investigation of the underlying tumor before it is clinically overt.

Paraneoplastic cerebellar degeneration (PCD) usually presents with an acute or a subacute onset of limb and trunk ataxia, dysarthria, dysphagia, diplopia and vertigo, and progresses rapidly within 3 months (6). A variety of malignancies are commonly associated with PCD, including small-cell lung cancer, Hodgkin's lymphoma, breast cancer and gynecological malignancies.

We herein report the case of a female patient with PCD caused by breast cancer and present a review of the literature on the mechanisms, clinical characteristics, diagnosis and management of this disorder.

\section{Case report}

A 67-year-old woman was admitted to the Department of Neurology of the First Affiliated Hospital of Nanjing Medical University (Nanjing, China) with a 6-month history of progressively worsening dizziness and unsteadiness while walking, accompanied by nausea and vomiting. The patient's past medical history was unremarkable. Cerebrospinal fluid (CSF) 
analysis revealed slightly elevated protein concentration with a normal cell count. A neurological workup at a different institution initially suggested a possible diagnosis of Miller Fisher syndrome. The patient was treated with immunoglobulins intravenously, with no significant neurological improvement. The patient gradually developed psychiatric symptoms, such as irritability and personality changes, followed by double vision over the next few months.

During hospitalization in our department, the patient's vital signs, including body temperature, heart rate, respiratory rate, blood pressure and oxygen saturation, were within normal limits. General physical examination, including breast examination, was normal. Neurological examination displayed dysarthria, bilateral gaze-evoked nystagmus, bilateral finger-nose ataxia, bilateral heel-shin ataxia, gait ataxia and positive bilateral Babinski sign. The results of routine laboratory tests were unremarkable and the serum tumor markers were within the normal range. Magnetic resonance imaging (MRI) of the brain did not reveal any remarkable abnormalities, apart from mild lacunar infarctions. The CSF analysis revealed slightly increased protein level, without oligoclonal bands. Electroencephalography revealed mild background slowing activity.

The initial diagnostic hypothesis was Wernicke's encephalopathy; however, there was no clinical improvement of the cerebellar symptoms despite the administration of thiamine. After excluding other potential causes of the neurological symptoms, a suspicion of PCD was raised. The serum was tested for characterized onconeural antibodies, including anti-Yo, anti-Hu, anti-Ri, anti-CV2, anti-Ma2 and anti-amphiphysin. A high titer of anti-Yo antibodies $(1: 1,000)$ was detected by indirect immunofluorescence assay and western blot analysis in the serum of the patient, which raised a high suspicion of breast and/or gynecological malignancy. Immunofluorescence assay and western blot analysis was conducted by EUROIMMUN Medical Diagnostics (China) Co., Ltd., (Beijing, China). Membrane strips containing electrophoretically-separated antigen extracts served as the solid phase. In the first incubation step, the specific antibodies in the diluted serum sample attach to the antigens coupled to the solid phase at room temperature for $30 \mathrm{~min}$. In the second incubation step, the bound antibodies were incubated with AP-labelled anti-human antibodies (ab97162; 1:10,000; Abcam Cambridge, UK) at room temperature for $30 \mathrm{~min}$. In a third step, the bound antibodies were stained with a chromogen/substrate solution capable of inducing a chromogenic reaction. An intense dark band at the line of the corresponding antigen appears if the serum sample contains specific antibodies. The intensity of the bands was automatically evaluated by the EUROLineScan program [EUROIMMUN Medical Diagnostics (China) Co., Ltd.]. Immunofluorescence was conducted using primate cerebellum and gut substrate [EUROIMMUN Medical Diagnostics (China) Co., Ltd.] as antigen substrates. Physically- or chemically- activated cover glasses coated with antigens were used as a solid phase. The specific antibodies in the diluted serum samples were incubated with the antigens coupled to a solid phase at room temperature for $30 \mathrm{~min}$. Subsequently, the samples were incubated with fluorescein-labelled anti-human $\operatorname{IgG}$ secondary antibodies (ab6584; 1:500, Abcam) at room temperature for
30 min and visualized under a fluorescent microscope with an excitation wavelength of $493 \mathrm{~nm}$. A whole-body positron emission tomography/computed tomography (PET/CT) scan revealed a $2.4 \times 1.3-\mathrm{cm}$ lesion in the lower outer quadrant of the left breast with slightly increased ${ }^{18} \mathrm{~F}$-fluorodeoxyglucose (FDG) uptake, as well as enlarged axillary and subclavian lymph nodes with mild FDG accumulation. Mammography revealed a lobulated high-density mass in the left breast (Fig. 1). Subsequently, an ultrasound-guided breast biopsy was performed and the results of the histopathological examination revealed a ductal carcinoma. The patient underwent left breast lumpectomy, and the diagnosis following immunohistological examination was grade II invasive ductal carcinoma, negative for estrogen and progesterone receptor, and positive for HER-2 and Ki-67 (Fig. 2). Pathologically, the tumor was classified as T2N0M0, stage IIA. After breast surgery, the psychiatric symptoms had improved at the 3-month follow-up, and the cerebellar symptoms, including dizziness and unsteadiness while walking, exhibited marked improvement at the 6- and 12-month follow-up visits. Overall, the patient exhibited a marked neurological improvement at the 12-month follow-up.

\section{Discussion}

PCD is one of the most common subtypes of PNS (3). Severe cerebellar symptoms with ataxia, dysarthria, dysphagia, vertigo, diplopia and nystagmus may appear abruptly or subacutely. The neurological syndrome progresses over weeks to months and then stabilizes on a very poor functional level, with patients unable to walk or perform fine motor movements.

In the early stages of the disease, the findings on brain MRI are usually normal, but cerebellar atrophy may develop later during the course of the disease (7). FDG-PET may reveal either hypometabolism or hypermetabolism, which represent cerebellar neuronal cell loss or an associated inflammatory response, respectively (8). CSF analysis usually shows mild lymphocytic pleocytosis and increased protein content, along with other signs of inflammatory or autoimmune disease (7). Autopsy reports show cerebellar atrophy with extensive loss of Purkinje cells in the cerebellar cortex, gliosis and inflammatory infiltrates (9).

Several autoantibodies associated with PCD have been reported, including anti-Yo, anti-Hu, anti-Tr, anti-voltage-gated calcium channel, anti-Ri and antiCV2 (10). Anti-Yo antibodies are most frequently detected in patients with cerebellar degeneration and breast or gynecological cancers (7). A total of 62 cases of PCD were eventually identified and fully characterized between 1966 and 1990, of which 19 patients were anti-Yo-positive (11). Recently, several PCD cases with positive anti-Yo antibody have been reported (12). Anti-Yo antibodies target the Purkinje cells of the cerebellum, resulting in extensive Purkinje cell death. The underlying mechanism is generally considered to be an immunological response to cerebellar degeneration-related protein 2, which is ectopically expressed by tumor cells (13). The presence of the autoantibodies in the serum of our patient raised a high suspicion of a breast and/or gynecological malignancy. The diagnostic studies, including radiological evaluation and histological examination, confirmed the 

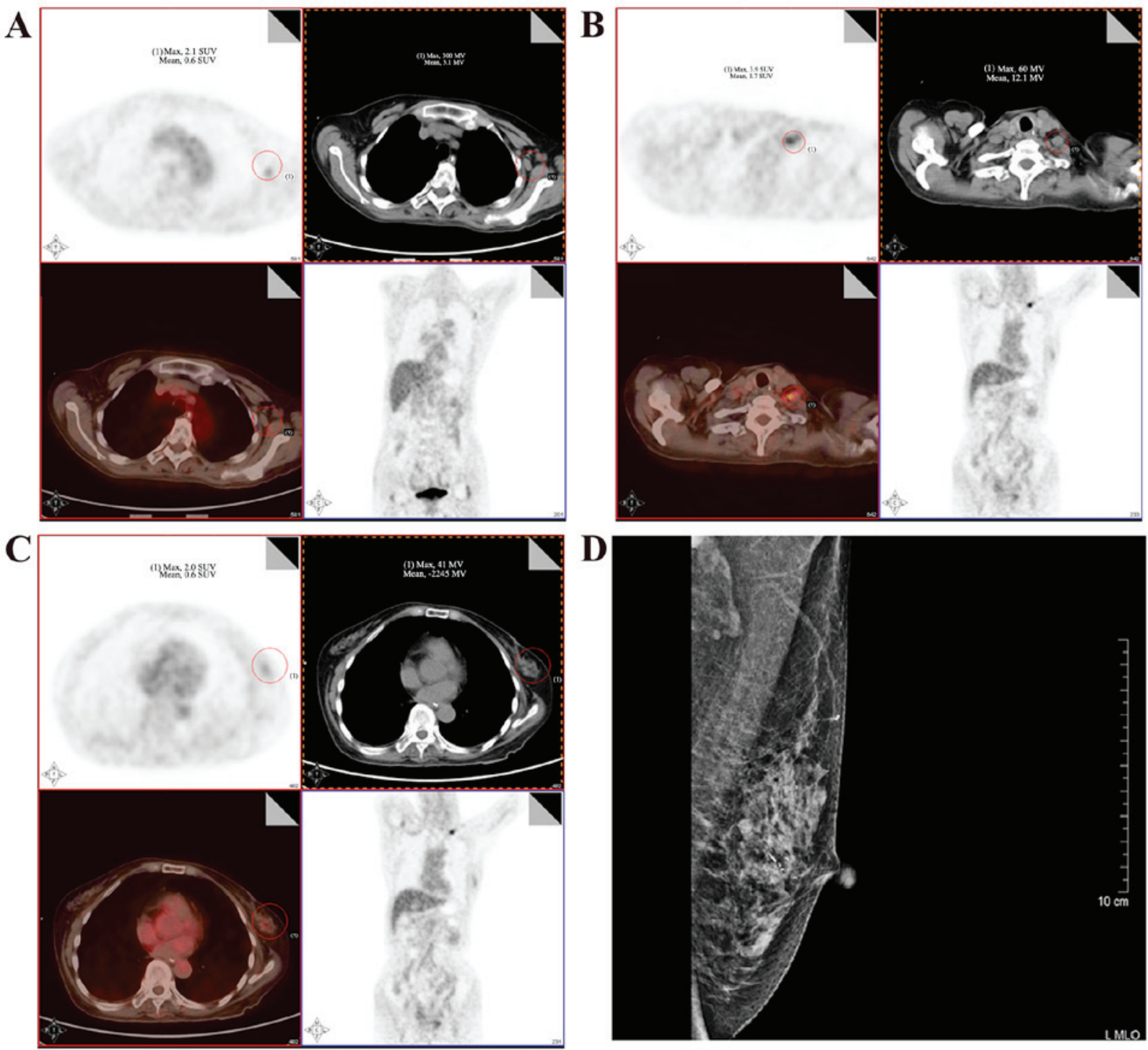

Figure 1. ${ }^{18} \mathrm{~F}$-Fluorodeoxyglucose (FDG) positron emission tomography/computed tomography (PET/CT) scan and the mammogram of the patient. (A) ${ }^{18} \mathrm{~F}$-FDG-PET/CT scan showing intense hypermetabolism at the level of the enlarged left axillary lymph nodes, with a maximum standardized uptake value $\left(\mathrm{SUV}_{\max }\right)$ of 3.6. (B) Hypermetabolism at the level of the enlarged left subclavian lymph nodes $\left(\mathrm{SUV}_{\max }, 4.9\right)$. (C) Lesion with uneven density in the lower outer quadrant of the left breast $\left(\mathrm{SUV}_{\max }, 2.0\right)$. (D) Mammography revealed the presence of a lobulated high-density mass in the left breast. The red circles indicate the lesion.

presence of breast cancer and allowed for early clinical management of the patient.

According to the diagnostic criteria for PNS proposed by an international panel of neurologists in 2004, two levels of evidence are suggested to define such a neurological syndrome: 'Definite' and 'possible' (14). The diagnostic criteria for definite PNS include a classical syndrome with well-characterized onconeural antibodies (anti-Yo, Hu, Ri, CV2, Ma2, or amphiphysin), or cancer that occurs within 5 years of the diagnosis of neurological symptoms. The term 'classic' syndromes refers to those neurological syndromes that are often associated with cancer. A clinical analysis of 55 anti-Yo antibody-positive patients demonstrated that, in the majority of the patients, the onset of PCD precedes the tumor diagnosis by months or even years (7); occasionally, carcinomas remain occult and are only detected on autopsy. The identification of a classic syndrome should prompt the screening for an underlying tumor. The panel defines the cerebellar syndrome as a classic syndrome based on the following criteria: Development of a severe cerebellar syndrome within 12 weeks, with no evidence of cerebellar atrophy on neuroradiological imaging, other than what would be expected by the patient's age. After excluding other known causes of the neurological syndrome, PNS should be considered in the differential diagnosis by the clinicians. Paraneoplastic antibodies should be detected in the serum or CSF, followed by a search for a primary tumor. PET/CT scans play a key role in the screening for the underlying cancer (15). Our patient exhibited characteristics of a classic cerebellar syndrome. Along with the identification of anti-Yo antibodies and breast cancer, the present case meets the diagnostic criteria for definite PCD.

The limited clinical trials for treatment strategies have made it difficult to establish evidence-based therapeutic guidelines. Currently, the mainstream principles of treatment include oncological therapy and immunotherapy, which are empirically based on reports from cases series and 

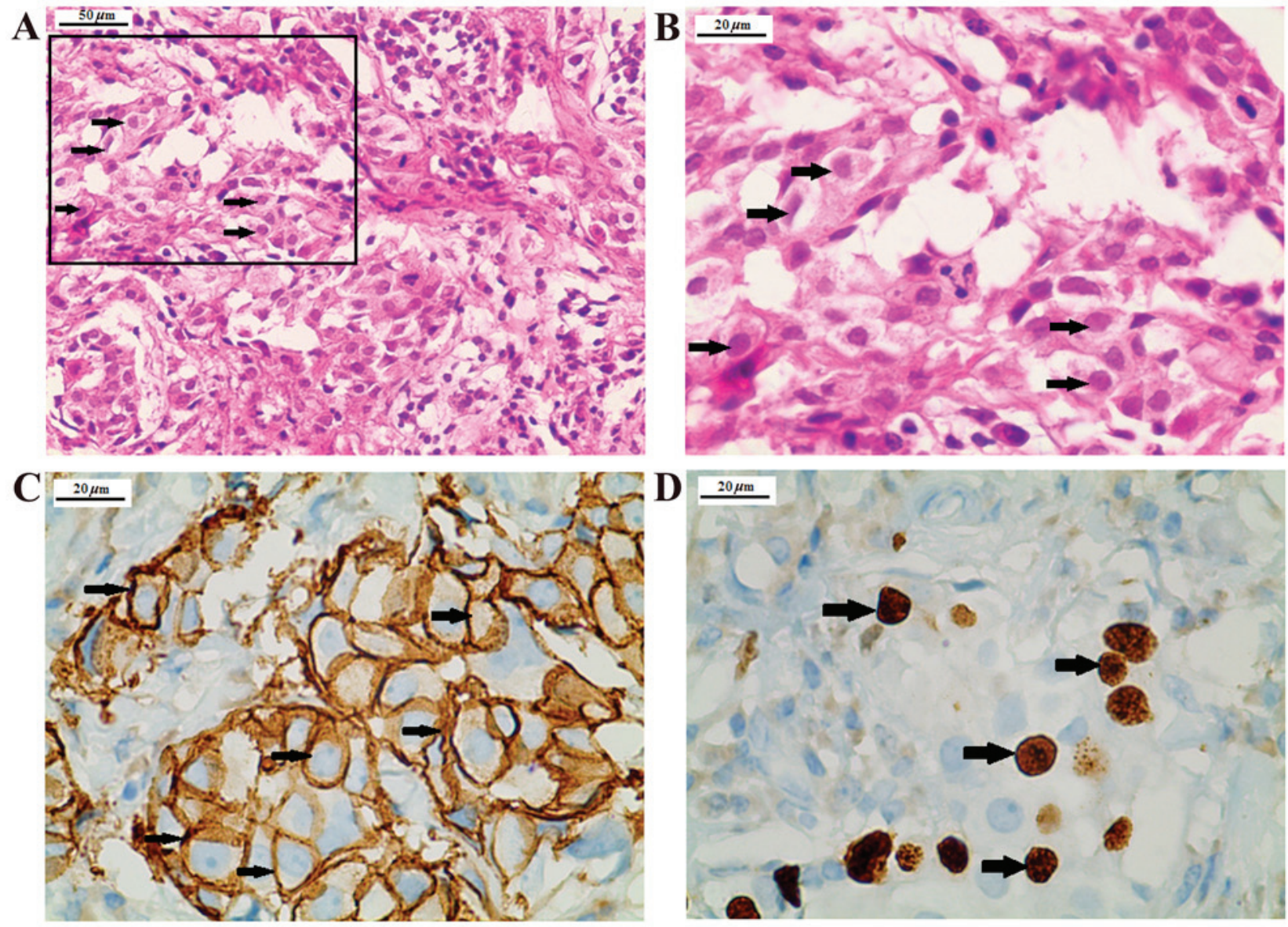

Figure 2. Histopathological and immunostaining analysis of the breast lumpectomy specimen. (A) Hematoxylin and eosin staining showing a ductal carcinoma. Scale bar, $50 \mu \mathrm{m}$. (B) Magnification of the inset in A. (C) Positive immunoreactivity for HER-2. (D) Positive immunoreactivity for Ki-67. Scale bar, $20 \mu \mathrm{m}$. HER-2, human epidermal growth factor receptor-2.

cohort studies (16). Early identification of the tumor and timely intervention enables stabilization or improvement of the neurological disorders. Patients receiving oncological therapy live significantly longer, regardless of the use of immunotherapy (10). Immunosuppression or immunomodulation are crucial components of the treatment of PNS. Initial treatment options often include corticosteroids, intravenous immunoglobulin (IVIG) and plasma exchange. Second-line immunosuppression, including cyclophosphamide, cyclosporine and rituximab, may be used when there is no apparent improvement with initial treatments. It has been suggested that high doses of IVIG and steroids may be helpful when used in the early stage of PCD (17). Another case series reported that a combination of IVIG, cyclophosphamide and methylprednisolone may stabilize the neurological condition in a minority of patients who are not severely disabled (18). Our patient exhibited marked clinical and neurological improvement after the treatment of breast cancer.

In summary, PCD is a rare neurological complication of cancer and often precedes the diagnosis of the underlying tumor. We herein report the case of a patient with PCD who had been misdiagnosed prior to the detection of the anti-Yo autoantibodies, and was eventually diagnosed with breast cancer. The presence of characterized anti-onconeural antibodies in the serum or CSF can provide guidance in the search for an underlying tumor. Prompt identification and treatment of the tumor may help stabilize or improve the neurological disorder.

\section{Acknowledgements}

Not applicable.

\section{Funding}

The present study was supported by the National Natural Science Foundation of China (grant no. 81600970) and the Project Funded by the Priority Academic Program Development of Jiangsu Higher Education Institutions (PAPD).

\section{Availability of data and materials}

Not applicable.

\section{Authors' contributions}

LY, HX, JH and WW analyzed and interpreted the patient data. XD was a major contributor in writing the manuscript. LH, QW were involved in revising the manuscript critically for important intellectual content. JG gave final approval of the version to be published. The final version of the manuscript has been read and approved by all authors. 


\section{Ethics approval and consent to participate}

The present study was approved by the Ethics Committee of the First Affiliated Hospital of Nanjing Medical University, Nanjing, China.

\section{Consent for publication}

Written informed consent was obtained from the patient for the publication of this case report and accompanying images.

\section{Competing interests}

The authors declare that they have no competing interests.

\section{References}

1. Höftberger R, Rosenfeld MR and Dalmau J: Update on neurological paraneoplastic syndromes. Curr Opin Oncol 27: 489-495, 2015.

2. Braik T, Evans AT, Telfer M and McDunn S: Paraneoplastic neurological syndromes: Unusual presentations of cancer. A practical review. Am J Med Sci 340: 301-308, 2010.

3. Giometto B, Grisold W, Vitaliani R, Graus F, Honnorat J and Bertolini G; PNS Euronetwork: Paraneoplastic neurologic syndrome in the PNS Euronetwork database: A European study from 20 centers. Arch Neurol 67: 330-335, 2010.

4. Graus F, Saiz A and Dalmau J: Antibodies and neuronal autoimmune disorders of the CNS. J Neurol 257: 509-517, 2010.

5. Titulaer MJ, Soffietti R, Dalmau J, Gilhus NE, Giometto B, Graus F, Grisold W, Honnorat J, Sillevis Smitt PA, Tanasescu R, et al: Screening for tumours in paraneoplastic syndromes: Report of an EFNS task force. Eur J Neurol 18 : 19-e23, 2011.

6. Venkatraman A and Opal P: Paraneoplastic cerebellar degeneration with anti-Yo antibodies: a review. Ann Clin Transl Neurol 3: 655-663, 2016.

7. Peterson K, Rosenblum MK, Kotanides $\mathrm{H}$ and Posner JB: Paraneoplastic cerebellar degeneration. I. A clinical analysis of 55 anti-Yo antibody-positive patients. Neurology 42: 1931-1937, 1992.

8. Mascalchi M and Vella A: Magnetic resonance and nuclear medicine imaging in ataxias. Handb Clin Neurol 103: 85-110, 2012.

9. Verschuuren J, Chuang L, Rosenblum MK, Lieberman F, Pryor A, Posner JB and Dalmau J: Inflammatory infiltrates and complete absence of Purkinje cells in anti-Yo-associated paraneoplastic cerebellar degeneration. Acta Neuropathol 91: 519-525, 1996
10. Shams'ili S, Grefkens J, de Leeuw B, van den Bent M, Hooijkaas H, van der Holt B, Vecht C and Sillevis Smitt P: Paraneoplastic cerebellar degeneration associated with antineuronal antibodies: Analysis of 50 patients. Brain 126: 1409-1418, 2003.

11. Fanous I and Dillon P: Paraneoplastic neurological complications of breast cancer. Exp Hematol Oncol 5: 29, 2016.

12. Adama D, Moussa B, Emmanuel M and Dennis U: Breast cancer revealed by a paraneoplastic cerebellar syndrome: About one case and literature review. Pan Afr Med J 22: 25, 2015.

13. Jarius S and Wildemann B: 'Medusa head ataxia': The expanding spectrum of Purkinje cell antibodies in autoimmune cerebellar ataxia. Part 3: Anti-Yo/CDR2, anti-Nb/AP3B2, PCA-2, anti-Tr/DNER, other antibodies, diagnostic pitfalls, summary and outlook. J Neuroinflammation 12: 168, 2015.

14. Greenlee JE: Recommended diagnostic criteria for paraneoplastic neurological syndromes. J Neurol Neurosurg Psychiatry 75: 1090, 2004.

15. Pena Pardo FJ, García Vicente AM, Amo-Salas M, López-Fidalgo JF, Garrido Robles JA, de Ayala Fernández JÁ, Del Saz Saucedo P, Muñoz Pasadas M and Soriano Castrejón A: Utility of 18F-FDG-PET/CT in patients suspected of paraneoplastic neurological syndrome: importance of risk classification. Clin Transl Oncol 19: 111-118, 2017.

16. Vedeler CA, Antoine JC, Giometto B, Graus F, Grisold W, Hart IK, Honnorat J, Sillevis Smitt PA, Verschuuren JJ and Voltz R; Paraneoplastic Neurological Syndrome Euronetwork: Management of paraneoplastic neurological syndromes: Report of an EFNS Task Force. Eur J Neurol 13: 682-690, 2006.

17. Widdess-Walsh P, Tavee JO, Schuele S and Stevens GH: Response to intravenous immunoglobulin in anti-Yo associated paraneoplastic cerebellar degeneration: Case report and review of the literature. J Neurooncol 63: 187-190, 2003.

18. Keime-Guibert F, Graus F, Fleury A, René R, Honnorat J, Broet P and Delattre JY: Treatment of paraneoplastic neurological syndromes with antineuronal antibodies (Anti-Hu, anti-Yo) with a combination of immunoglobulins, cyclophosphamide, and methylprednisolone. J Neurol Neurosurg Psychiatry 68: 479-482, 2000 .

(i) $\Theta$ This work is licensed under a Creative Commons Attribution-NonCommercial-NoDerivatives 4.0 International (CC BY-NC-ND 4.0) License. 